\title{
Development of a three-dimensional, unstructured material response design tool
}

\author{
Joseph C. Schulz* Eric C. Stern ${ }^{\dagger} \quad$ Suman Muppidi* $\quad$ Grant E. Palmer * \\ Olivia Schroeder $\ddagger$ \\ Analytical Mechanic Associates, Inc. \\ NASA Ames Research Center, Moffett Field, CA
}

\begin{abstract}
A preliminary verification and validation of a new material response model is presented. This model, Icarus, is intended to serve as a design tool for the thermal protection systems of re-entry vehicles. Currently, the capability of the model is limited to simulating the pyrolysis of a material as a result of the radiative and convective surface heating imposed on the material from the surrounding high enthalpy gas. Since the major focus behind the development of Icarus has been model extensibility, the hope is that additional physics can be quickly added. This extensibility is critical since thermal protection systems are becoming increasing complex, e.g. woven carbon polymers. Additionally, as a three-dimensional, unstructured, finite-volume model, Icarus is capable of modeling complex geometries. In this paper, the mathematical and numerical formulation is presented followed by a discussion of the software architecture and some preliminary verification and validation studies.
\end{abstract}

\section{Nomenclature}

$c_{v} \quad$ Total specific heat per unit mass $\left[\mathrm{J} \cdot \mathrm{kg}^{-1}\right]$

$c_{v, g} \quad$ Specific heat at constant volume of the gas mixture per unit mass $\left[\mathrm{J} \cdot \mathrm{kg}^{-1}\right]$

$c_{v, s} \quad$ Specific heat of the material per unit mass $\left[\mathrm{J} \cdot \mathrm{kg}^{-1}\right]$

$e \quad$ Total energy per unit mass $\left[\mathrm{J} \cdot \mathrm{kg}^{-1}\right]$

$e_{c} \quad$ Energy of the solid char products per unit mass $\left[\mathrm{J} \cdot \mathrm{kg}^{-1}\right]$

$e_{g} \quad$ Energy of the gas mixture per unit mass $\left[\mathrm{J} \cdot \mathrm{kg}^{-1}\right]$

$e_{s} \quad$ Energy of the material per unit mass $\left[\mathrm{J} \cdot \mathrm{kg}^{-1}\right]$

$e_{v} \quad$ Energy of the virgin material per unit mass $\left[\mathrm{J} \cdot \mathrm{kg}^{-1}\right]$

$h \quad$ Total enthalpy per unit mass $\left[\mathrm{J} \cdot \mathrm{kg}^{-1}\right]$

$h_{c} \quad$ Enthalpy of the solid char products per unit mass [ $\left.\mathrm{J} \cdot \mathrm{kg}^{-1}\right]$

$h_{g} \quad$ Enthalpy of the gas mixture per unit mass $\left[\mathrm{J} \cdot \mathrm{kg}^{-1}\right]$

$h_{s} \quad$ Enthalpy of the material per unit mass $\left[\mathrm{J} \cdot \mathrm{kg}^{-1}\right]$

$h_{v} \quad$ Enthalpy of the virgin material per unit mass $\left[\mathrm{J} \cdot \mathrm{kg}^{-1}\right]$

$p \quad$ Pressure of the gas mixture $[\mathrm{Pa}]$

$T_{a, n}$ Arrhenius activation temperature for the $n$-th pyrolysis reaction $[\mathrm{K}]$

$T$ Temperature of the gas and material $[\mathrm{K}]$

$t \quad$ Time [sec]

$u_{g, i} \quad$ Velocity of the pyrolysis gas mixture $\left[\mathrm{m} \cdot \mathrm{sec}^{-1}\right]$

$W_{g} \quad$ Molecular weight of the pyrolysis gas mixture $\left[\mathrm{kmol} \cdot \mathrm{kg}^{-1}\right]$

$x_{i} \quad$ Cartesian coordinates within simulation domain [m]

$Y_{v} \quad$ Mass fraction of virgin material

$\beta \quad$ Extent of the pyrolysis or fraction of charred material

*AMA, Inc., Moffett Field, CA, 94035, USA

$\dagger$ NASA Ames Research Center, Moffett Field, CA, 94035, USA

${ }^{\ddagger}$ Gradudate Researcher, University of Kentucky 


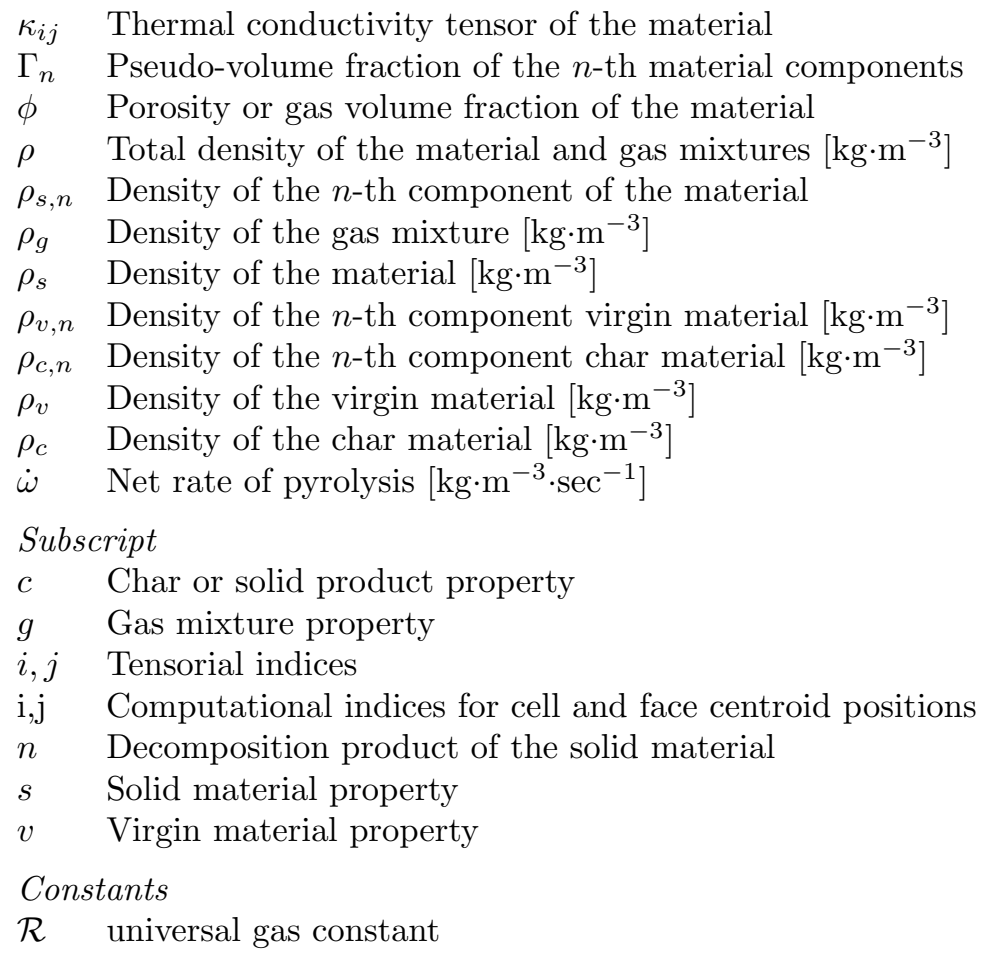

\section{Introduction}

During spacecraft re-entry, the high enthalpy gas surrounding the vehicle transfers a fraction of the dissipated kinetic energy by radiative and convective heating to the surface of the vehicle. As the temperature within the material increases, two physical processes, pyrolysis and ablation, alter the mechanical properties and chemical composition of the material. Controlling this material response to within design limits is the objective of the thermal protection system (TPS). At a minimum the design of the TPS depends on two parameters, the ablation rate and the peak temperature at the bondline between the material and the substructure. The objective of a material response tool is to predict these quantities in order to estimate the required TPS material thickness. This objective drives the requirements for development of any new, design focused, material response model.

Depending on the TPS and the type material used, the relative importance of the different physical mechanisms occurring during pyrolysis and ablation dictate the complexity of the material response design tool. Viewed from this perspective, a hierarchy of material response model fidelity has recently been proposed to aid in understanding of how modeling assumptions could affect design decisions. ${ }^{1}$ The hierarchy is based on the types of physical mechanisms occurring within the material and is used to categorize the modeling capability of several current material response codes.

Given the potential need to design the TPS for missions of increasing entry speed and heat load, there is a need for a material response design tool to have varying degrees of model fidelity. Additionally, a numerical method based on an unstructured finite volume approximation increases the flexibility in simulating complex surface geometries. In this paper, the mathematical formulation is first presented and is followed by a description of the numerical method. Preliminary results are then presented for verification of the implementation. Lastly, guidance on future work to be included in the final manuscript is provided.

\section{Formulation}

\section{II.A. Governing Equations}

Pyrolysis results in the decomposition of the virgin material into gaseous and solid products. For many TPS materials, the process is so complex that simplistic reduced-order models are still widely used for engineering design. For example, in scenarios where the solid products are highly porous, the product gases easily diffuse, 
convect, and mix with other gases within the material resulting in an ever increasing set of reactions. While there is interest in modeling a more complete set of finite-rate kinetics for pyrolysis, the current formulation adopts the traditional approach of using engineering models.

Since pyrolysis is a decomposition process, it is essentially a mass balance between the initial virgin material and two products, a decomposed solid or liquid and a gas mixture. If the products are in local chemical and thermal equilibrium (at least on the time scale of thermal diffusion), then the number of distinct product components and the number of separate mass and energy balance equations can be reduced. In this simplified approach, the decomposition of the solid virgin material can be modeled through a set of $N$ pyrolysis reactions involving $N$ solid components and a single gas mixture. The total density of the material is computed based on the initial pseudo-volume fraction, $\Gamma_{n}$, of the solid material components. Hence,

$$
\rho_{s}=\sum_{n} \Gamma_{n} \rho_{s, n}
$$

where $\Gamma_{n}$, as a pseudo-volume fraction does not necessarily sum to 1 . Based on this assumption, the pyrolysis gas mixture occupies the entire pore volume of the solid decomposition products. If $\phi$ is the material porosity, then the total density of the solid and gas mixture is

$$
\rho=\phi \rho_{g}+\rho_{s}
$$

where $\rho_{s}$ is the bulk density of the solid, and $\phi \rho_{g}$ is the bulk density of pyrolysis gas mixture. In this interpretation, the gas density, $\rho_{g}$, is a true or particle density, and the porosity, $\phi$, is equal to the gas volume fraction.

If the decomposition rates follow an Arrhenius form, then the time rate of change for each of the solid material components is given as

$$
\frac{\partial \rho_{s, n}}{\partial t}=-k_{n} \rho_{v, n}\left(\frac{\rho_{s, n}-\rho_{c, n}}{\rho_{v, n}}\right)^{\psi_{n}} \mathrm{e}^{\left(-T_{a, n} / T\right)}, \quad n=1, \ldots, N
$$

where $k_{n}, \psi_{n}$, and $T_{a, n}$ are the Arrhenius reaction rate, pre-exponential, and activation temperature of the pyrolysis reaction related to the $n$ component of the material, respectively. The reactions are all irreversible, and the net rate is by definition proportional to the production of pyrolysis gases. Thus, the pyrolysis rate times the pseudo-volume fraction is equal to the net production of pyrolysis gas,

$$
\dot{\omega}=\sum_{n}^{N} \Gamma_{n} \frac{\partial \rho_{s, n}}{\partial t} .
$$

As an example, in a widely used three-component decomposition model, ${ }^{2}$ the virgin material contains a phenolic resin, which undergoes a two-stage decomposition process, and a binder composite, which undergoes a single-stage decomposition. Thus, the bulk solid density is $\rho_{s}=\Gamma\left(\rho_{A}+\rho_{B}\right)+(1-\Gamma) \rho_{C}$, where $\rho_{A}$ and $\rho_{B}$ represent the components of the phenolic resin and $\rho_{C}$ represents the binder composite. ${ }^{3}$ Yet, it is important to note that the notion of three distinguishable species $A, B$, and $C$ is an artifact of the model.

In addition to the total mass and energy conservation of the material, the pyrolysis gases must satisfy additional mass and momentum conservation equations. With the production rate of pyrolysis gases as $\dot{\omega}$ and the diffusive/convective velocity of the gases through the material as $u_{g, i}$, the mass conservation equation for the gas mixture is

$$
\frac{\partial\left(\phi \rho_{g}\right)}{\partial t}+\frac{\partial}{\partial x_{i}}\left(\phi \rho_{g} u_{g, i}\right)=\dot{\omega},
$$

where $\phi \rho_{g}$ is the bulk density of pyrolysis gas mixture and is the conserved quantity. For porous flows, the velocity of the pyrolysis gas, $u_{g, i}$, can be approximated from Darcy's law, a simplified form of the momentum conservation equation that states that the velocity of the gas is proportional to pressure gradient within the material. Mathematically, this states

$$
u_{g, i}=-\frac{1}{\mu} K_{i j} \frac{\partial p}{\partial x_{j}},
$$

where $\mu$ is the viscosity of the gas mixture, and $K_{i j}$ is the permeability tensor. For isotropic materials, the permeability reduces to a scalar quantity. 
To complete the set of governing equations, a total energy conservation equation for the solid and gas mixture is necessary. For a material undergoing only pyrolysis, the transfer of energy occurs through the convection of the pyrolysis gas and thermal conduction within the material, which is assumed to obey Fourier's law. Like the permeability, since the material can be anisotropic, the thermal conductivity is a tensor, $\kappa_{i j}$. In the absence of radiative energy transfer, the complete set of conservation equations along with the energy conservation equation is given by

$$
\begin{aligned}
& \frac{\partial \rho_{s, n}}{\partial t}=-k_{n} \rho_{v, n}\left(\frac{\rho_{k}-\rho_{c, n}}{\rho_{v, n}}\right)^{\psi_{n}} \mathrm{e}^{\left(-T_{a, n} / T\right)}, \\
& \frac{\partial\left(\phi \rho_{g}\right)}{\partial t}+\frac{\partial}{\partial x_{i}}\left(\phi \rho_{g} u_{g, i}\right)=\dot{\omega}, \\
& \frac{\partial(\rho e)}{\partial t}+\frac{\partial}{\partial x_{i}}\left(\phi \rho_{g} h_{g} u_{g, i}\right)-\frac{\partial}{\partial x_{i}}\left(\kappa_{i j} \frac{\partial T}{\partial x_{j}}\right)=0 .
\end{aligned}
$$

In order to complete the set of governing equations, thermodynamic and transport models are necessary. The next two sections describe the choice of thermodynamic state variables as well as how the transport properties are computed within a material and gas mixture.

\section{II.B. State Variables}

A result of using a macroscopic model for the pyrolysis is an incomplete knowledge of the time-varying elemental and molecular composition. As a result, the state of the material must be linearly interpolated between their virgin state and their fully charred state. Only at these two states are the TPS materials measured. This interpolation uses the extent of reaction or the fraction of charred material, which is defined as

$$
\beta=\frac{\rho_{v}-\rho_{s}}{\rho_{v}-\rho_{c}},
$$

where $\rho_{v}$ and $\rho_{c}$ are the measured bulk densities of the solid virgin and solid product material or char, respectively. This definition can be re-arranged into a definition for the virgin mass fraction,

$$
Y_{v}=\frac{\rho_{v}}{\rho_{v}-\rho_{c}}\left(1-\frac{\rho_{c}}{\rho_{s}}\right),
$$

which is related to the extent of reaction by

$$
Y_{v}=\left(\frac{\rho_{v}}{\rho_{s}}\right)(1-\beta) .
$$

Similarly, the mass fraction of the solid char is given as $Y_{c}=1-Y_{v}=\left(\rho_{c} / \rho_{s}\right) \beta$. Once again, the fictitious notion of virgin and char material components is an artifact relying on the available thermophysical data. A more exact model would require modeling the time-varying elemental and molecular composition of the material. ${ }^{1}$

The physical properties of the material are either available in the form of a table or as a curve-fitted function at both the virgin and charred states. The internal energies and specific heats are a function of temperature and possibly the internal pressure of the gas. The mixture-averaged properties are determined as a linear combination of the virgin or char mass values. For instance, the internal energy of the solid material is given by

$$
e_{s}(p, T)=h_{s}(p, T)=Y_{v} e_{v}(p, T)+\left(1-Y_{v}\right) e_{c}(p, T),
$$

where $e_{s}$ and $h_{s}$ are the mixture-averaged energy (enthalpy) of the solid material. A similar expression is used to compute the mixture-averaged specific heat $c_{v, s}$ of the solid material.

The thermodynamic state of the pyrolysis gas is described by the perfect gas law. Assuming that the solid and gas phases are in equilibrium, the pressure of the pyrolysis gas mixture is

$$
p=\rho_{g} \frac{\mathcal{R}}{W_{g}} T,
$$


where $\mathcal{R}$ is the universal gas constant, $W_{g}$ is the molecular weight of the gas mixture, and $T$ is the equilibrium temperature of the solid and gas components. The gas mixture specific heat, energy, and enthalpy are computed from temperature dependent curve-fitted expressions provided by the NASA Chemical Equilibrium with Applications (CEA) database. ${ }^{4}$ These curve-fits are provided for each constituent species of the gas and then averaged using the equilibrium species mass fractions to compute the total mixture-averaged gas quantities. In addition to the option of using the CEA curve-fits, either the Cantera or the Mutation++ library can be used to compute these quantities directly. More discussion about these options are provided in a later section.

Defining the gas mass fraction as $Y_{g}=\phi \rho_{g} / \rho$, the total energy and enthalpy of the combined solid and gas phases of the material are given as

$$
e(T)=Y_{g} e_{g}(T)+\left(1-Y_{g}\right) e_{s}(T)
$$

and

$$
h(T)=Y_{g} h_{g}(T)+\left(1-Y_{g}\right) h_{s}(T),
$$

where $e_{g}$ and $h_{g}$ are the mixture-averaged energy and enthalpy of the pyrolysis gas, and $e$ and $h$ are the total energy and enthalpy of the combined solid and gas mixtures.

These thermodynamic state models provide the relationship between the temperature and pressure of the solid/gas mixture and the conserved mass and energy variables governed by the set of equations presented in the previous section. Since the material and gas are in chemical and thermodynamic equilibrium, the mixture properties of the gas are defined completely by the temperature and pressure state variables. Yet, the mixture-averaged properties of the gas do depend implicitly on the gas species composing the mixture. Hence, whenever the temperature and pressure of the system change, the equilibrium composition of gas mixture changes. This makes the relationship between the conserved quantities and the thermodynamic state variables more computationally involved.

Lastly, to complete the closure of the conservation equations presented in Sec. II.A, expressions for evaluating remaining material properties as well as the several transport coefficients of both the solid material and pyrolysis gas are needed. Like the thermodynamic properties, these transport properties of the material are only known experimentally in their virgin or fully charred state. Thus, they are evaluated in a fashion similar to the way the thermodynamic mixture quantities. A more detailed discussion is provided in the next section.

\section{II.C. Transport Properties}

The material properties are usually evaluated as bulk properties consisting of either the completely virgin material or the completely charred material. As a result, the influence of the pyrolysis gas on these properties is neglected.

From tabulated values of the virgin and fully charred material, the bulk thermal conductivity is evaluated as a linear function of the virgin mass fraction and the temperature. Since the thermal conductivity is not necessarily isotropic, tabular data is provided for the value of the thermal conductivities along each principle axis of the material. This requires specifying the tabular values for the thermal conductivity in-plane and through-plane directions,

$$
\begin{aligned}
& \hat{\kappa}_{\|}(T)=Y_{v} \hat{\kappa}_{\|, v}(T)+\left(1-Y_{v}\right) \hat{\kappa}_{\|, c}(T), \\
& \hat{\kappa}_{\perp}(T)=Y_{v} \hat{\kappa}_{\perp, v}(T)+\left(1-Y_{v}\right) \hat{\kappa}_{\perp, c}(T),
\end{aligned}
$$

where $\hat{\kappa}_{\perp,(v, c)}$ represents the through-plane component of the virgin or char material, and $\hat{\kappa}_{\|,(v, c)}$ represents the corresponding in-plane component. The thermal conductivity vector orientated along the principle axis of the material is defined as $\hat{\boldsymbol{\kappa}}=\left(\hat{\kappa}_{\|}, \hat{\kappa}_{\perp}, \hat{\kappa}_{\perp}\right)^{T}$. The thermal conductivity tensor in a general frame of reference, $\kappa_{i j}$ is then found by transforming the vector $\hat{\kappa}$ through a rotation matrix. More information about this transformation is provided in a later section.

The porosity and permeability properties of the pyrolysis gases are assumed to be independent of the gas temperature and are considered functions of only the extent of reaction, $\beta$. Tabular values are used for these quantities. Like the material thermal conductivity, the permeability can be specified as a tabular quantities in the in-plane and through-plane directions.

Lastly, the gas mixture viscosity, $\mu$, is either computed using Sutherland's law or computed using the Cantera or Mutation++ libraries. Additional material and/or transport properties are only required for 
boundary conditions accounting for gas surface interactions. In these cases, surface quantities such as the emissivity are required as well. While these values are provided to Icarus, this paper does not discuss them any further.

\section{Numerical Formulation}

\section{III.A. Finite Volume Formulation}

The set of governing equations Eqs. 7-9 can be compactly described in the vector form. In Cartesian coordinates, $\mathbf{x}^{T}=[x, y, z]$, the set of equations is

$$
\frac{\partial}{\partial t} \mathbf{q}(\mathbf{x}, t)+\frac{\partial}{\partial x}[\mathbf{f}(\mathbf{q}(\mathbf{x}, t))]+\frac{\partial}{\partial y}[\mathbf{g}(\mathbf{q}(\mathbf{x}, t))]+\frac{\partial}{\partial z}[\mathbf{h}(\mathbf{q}(\mathbf{x}, t))]=\dot{\mathbf{s}}(\mathbf{q}(\mathbf{x}, t))
$$

where $\mathbf{q}$ defines the set of conservative variables, and $\mathbf{f}$ and $\dot{\mathbf{s}}$ are the corresponding set fluxes and sources, respectively. In this form, the conservative variables are represented by the state vector

$$
\mathbf{q}^{T}=\left[\rho_{s, 1}, \ldots, \rho_{s, \mathrm{~N}}, \phi \rho_{g}, \rho e\right]
$$

A corresponding vector of primitive variables w, which contains the variables of density, temperature, velocity, etc., can be computed from the conservative variables and through the relations described in Sec. II.B. The fluxes $\mathbf{f}$ and $\mathbf{g}$ are given by

$$
\mathbf{f}=\left[\begin{array}{c}
0 \\
\vdots \\
0 \\
\phi \rho_{g} u_{g} \\
\phi \rho_{g} h_{g} u_{g}-\kappa_{x x} \frac{\partial T}{\partial x}-\kappa_{x y} \frac{\partial T}{\partial x}-\kappa_{x z} \frac{\partial T}{\partial x}
\end{array}\right], \quad \mathbf{g}=\left[\begin{array}{c}
0 \\
\vdots \\
0 \\
\phi \rho_{g} u_{g} \\
\phi \rho_{g} h_{g} u_{g}-\kappa_{y x} \frac{\partial T}{\partial y}-\kappa_{y y} \frac{\partial T}{\partial y}-\kappa_{y z} \frac{\partial T}{\partial y}
\end{array}\right],
$$

where the $z$-direction flux $\mathbf{h}$ follows similarly. The source term vector is

$$
\dot{\mathbf{s}}=\left[\begin{array}{cc}
-k_{1} \rho_{v, 1}\left(\frac{\rho_{s, 1}-\rho_{c, 1}}{\rho_{v, 1}}\right)^{\psi_{1}} & \mathrm{e}^{\left(-T_{a, 1} / T\right)} \\
\vdots & \\
-k_{\mathrm{N}} \rho_{v, \mathrm{~N}}\left(\frac{\rho_{s, \mathrm{~N}}-\rho_{c, \mathrm{~N}}}{\rho_{v, \mathrm{~N}}}\right)^{\psi_{\mathrm{N}}} & \mathrm{e}^{\left(-T_{a, \mathrm{~N}} / T\right)} \\
\dot{\omega} & \\
0 &
\end{array}\right]
$$

Using Gauss' theorem, the governing set of equations is converted to the integral form. In this form, and only considering a single dimension, the finite-volume representation of the governing equations is

$$
\frac{\partial}{\partial t} \int_{\mathcal{V}} \mathbf{q}(\mathbf{x}, t) \mathrm{d} \mathbf{x}+\int_{\mathcal{S}}(\mathbf{f}(\mathbf{q}(\mathbf{x}, t)) \cdot \mathbf{n}) \mathrm{d} \mathcal{S}=\int_{\mathcal{V}} \dot{\mathbf{s}}(\mathbf{q}(\mathbf{x}, t)) \mathrm{d} \mathbf{x} .
$$

where numerically this set of equations is solved by approximating the variables at discrete volume-averaged quantities separated by discrete time intervals, $\Delta t=t_{\mathrm{n}+1}-t_{\mathrm{n}}$. The cell-averaged conservative variables of the $\mathrm{i}$-th cell element at time $t_{\mathrm{n}}$ are defined as

$$
\mathbf{Q}_{\mathrm{i}}\left(t_{\mathrm{n}}\right) \approx \frac{1}{\mathcal{V}_{\mathrm{i}}} \int_{\mathcal{V}_{\mathrm{i}}} \mathbf{q}(\mathbf{x}, t) \mathrm{d} \mathbf{x}
$$

and the fluxes are defined as discrete averages over the cell area. Note that in the remaining sections, the Roman indices are used to represent computational points while the italicized indices are used for tensor and summation notation. 


\section{III.B. Explicit Time Integration}

In many situations relevant to the design of materials for thermal protection systems, explicit time integration of the governing equations usually does not impose significant time-stepping constraints on simulation. When they do appear, they occur in the evaluation of the pyrolysis chemical source source, which can be moderated in other ways. Hence, in the current formulation, an explicit time integration scheme of first- or second-order is used. Consider the first-order backward Euler time integration scheme. Advancing this set of equations in time results in the following numerical scheme,

$$
\mathbf{Q}_{\mathrm{i}}\left(t_{\mathrm{n}+1}\right)=\mathbf{Q}_{\mathrm{i}}\left(t_{n}\right)-\frac{1}{\mathcal{V}_{\mathrm{i}}} \sum_{\mathrm{j} \in J_{\mathrm{i}}}\left[\mathbf{F}_{\mathrm{i}, \mathrm{j}}\left(t_{\mathrm{n}}\right) \cdot \mathbf{n}_{\mathrm{i}, \mathrm{j}} \mathcal{S}_{\mathrm{i}, \mathrm{j}}\right] \Delta t+\dot{\mathbf{S}}_{\mathrm{i}}\left(t_{\mathrm{n}}\right) \Delta t
$$

where $J_{\mathrm{i}}$ represents the set of faces belonging to the $i$-th cell element where the surface area of each $\mathrm{j}$-th face on the i-th cell is $\mathcal{S}_{\mathrm{i}, \mathrm{j}}$. In general, using a higher order time advancement scheme follows the same principle and is easy to implement. For instance, a high order TVD and strong stability preserving (SSP) Runge-Kutta time integration scheme can be written in the general form ${ }^{5,6}$

$$
\begin{aligned}
& \mathbf{Q}^{(0)}=\mathbf{Q}^{\mathrm{n}} \\
& \mathbf{Q}^{(i)}=\sum_{k=0}^{i-1}\left(\alpha_{i k} \mathbf{Q}^{(k)}+\Delta t \beta_{i k} \mathcal{L}\left(\mathbf{Q}^{(k)}\right)\right) \quad i=1, \ldots, m \\
& \mathbf{Q}^{\mathrm{n}+1}=\mathbf{Q}^{m}
\end{aligned}
$$

where the spatial operator $\mathcal{L}$ represents some $r$-th order flux evaluation. The coefficients $\alpha_{i, k}$ and $\beta_{i, k}$ are non-negative and the maximum Courant-Friedrichs-Lewy $(\mathrm{CFL})^{7}$ is defined as

$$
C F L=\max _{i, k} \frac{\alpha_{i, k}}{\beta_{i, k}}
$$

For a two-stage, second-order SSP Runge-Kutta scheme, $\alpha_{1,1}=1, \alpha_{i, 2}=1 / 2, \beta_{1,1}=1$, and $\beta_{2,2}=1 / 2$. For a three-stage, third-order SSP Runge-Kutta scheme, $\alpha_{1,1}=1, \alpha_{1,2}=3 / 4, \alpha_{1,3}=1 / 3, \alpha_{2,2}=1 / 4$, $\alpha_{3,3}=2 / 3, \beta_{1,1}=1, \beta_{2,2}=1 / 4$, and $\beta_{2,2}=2 / 3$. Unless specified, all other values are zero. See references for more specific information. ${ }^{5,6}$

\section{III.C. Flux Evaulation}

In order to evaluate the fluxes, a method is required to compute quantities at the surface centroids using the volume-averaged cell-centered quantities. In this formulation, two methods are needed, one for the thermal diffusion fluxes and another for the convective fluxes. Beginning with the viscous or diffusion fluxes, quantities such as the temperature gradient are needed at the surface centroids of each computational cell. The simplest approximation is to use the Green-Gauss theorem to compute the discrete volume-averaged gradient at each cell element and then average them across the face to compute the face-averaged spatial derivative. The Green-Gauss theorem states,

$$
\int_{\mathcal{V}} \nabla \phi \mathrm{d} \mathcal{V}=\int_{\mathcal{S}} \phi \mathbf{n} \cdot \mathrm{d} \mathcal{S}
$$

where $\phi$ is a scalar quantity. In the discrete approximation, the volume-averaged scalar gradient at the i-th cell element, $\bar{\nabla}_{\mathrm{i}}$, is approximately equal to the summation of the scalar quantity, $\phi_{i}$, projected to each face of the cell. This results in the following approximation,

$$
\overline{\nabla \phi}_{\mathrm{i}}=-\frac{1}{\mathcal{V}_{\mathrm{i}}} \sum_{\mathrm{j} \in J_{\mathrm{i}}} \widehat{\phi}_{\mathrm{j}} \mathcal{S}_{\mathrm{i}, \mathrm{j}}
$$

where $\widehat{\phi}_{\mathrm{j}}$ is the value of the scalar at the face centroid of the $\mathrm{j}$-th face within the i-th cell. As a first-order approximation, $\widehat{\phi}_{\mathrm{j}}$ is computed as the mean of the volume-averaged quantities between the cells neighboring the $\mathrm{j}$-th face, $l$ and $r$. Thus, the face-averaged value is $\widehat{\phi}_{\mathrm{j}}=\left(\phi_{\mathrm{i}=l}+\phi_{\mathrm{i}=r}\right) / 2$. The face centroid gradients are 
computed by averaging the cell-centered gradients and applying correction term in order to avoid numerical oscillations resulting from odd-even decoupling. ${ }^{8}$ With this correction, the face-centered gradient is,

$$
\nabla \phi_{\mathrm{j}}=\frac{1}{2}\left(\bar{\nabla}_{\mathrm{i}=l}+\overline{\nabla \phi}_{\mathrm{i}=r}\right)-\widehat{\mathbf{d}}_{l r}\left(\frac{1}{2}\left(\overline{\nabla \phi}_{\mathrm{i}=l}+\overline{\nabla \phi}_{\mathrm{i}=r}\right) \cdot \widehat{\mathbf{d}}_{l r}\right)+\left(\phi_{\mathrm{i}=l}-\phi_{\mathrm{i}=r}\right) \frac{\widehat{\mathbf{d}}_{l r}}{\left|\mathbf{d}_{l r}\right|}
$$

where $\mathbf{d}_{l r}$ and $\widehat{\mathbf{d}}_{l r}$ are the distance and unit distance vector between the two cell-center indices, $l$ and $r$, neighboring the $\mathrm{j}$-th face, respectively.

For the convective fluxes, e.g., $\phi \rho_{g} u_{g, i}$, a first-order approximation is used compute the face-centered conservative variables, e.g., $\phi \rho_{g}$, needed to evaluate the flux. These values are directionally dependent on the sign of the pyrolysis gas velocity vector, $u_{g, i}$, which is evaulated at each $\mathrm{j}$-th face using Darcy's law,

$$
u_{g, i, \mathrm{j}}=-\frac{1}{\widehat{\mu}_{\mathrm{j}}} \widehat{K}_{i j, \mathrm{j}}\left(\frac{\partial p}{\partial x_{j}}\right)_{\mathrm{j}},
$$

where $\widehat{\mu}_{\mathrm{j}}$ and $\widehat{K}_{i j, \mathrm{j}}$ are the average gas viscosity and average permeability tensor at the $\mathrm{j}$-th face computed as $\widehat{\phi}_{\mathrm{j}}=\left(\phi_{\mathrm{i}=l}+\phi_{\mathrm{i}=r}\right) / 2$ where $l$ and $r$ are the cell center indices of the neighboring cells to the $\mathrm{j}$-th face, and $\phi$ is the scalar values of either the gas mixture viscosity or the components of the heat conduction tensor. In this first-order approximation, the cell-centered quantity $(\mathrm{i}=l$ or $\mathrm{i}=r)$ in the direction opposite of $\mathbf{u}_{g, i} n_{i, \mathrm{j}}$ at the face is used to approximate the face-center conservative values.

\section{III.D. Tensor Properties}

The materials used for thermal protection systems often exhibit non-isotropic behavior. For example, in many TPS materials, the thermal conductivity is lower in the through-thickness direction as compared to the in-plane direction. For TPS materials constructed by 'carving' out the material from a large block, the material is likely to be oriented by a global principal axis. Similarly, if the TPS material is tiled, then each tile could have its own global principal axis. In other cases, defining a global principle axis for the material is not straight forwared. For instance, woven TPS materials have orthotropic properties whose principal axes vary along contour of the body. For numerical computations, this requires tracking the orientation of the principal axis at each face centroid.

Thermal conductivity for orthotropic materials is expressed in terms of two scalar quantities, $\hat{\kappa}_{\|}$and $\hat{\kappa}_{\perp}$. If the Cartesian directions align with the principal axes of the material, the conductivity tensor looks like

$$
\kappa_{i j}=\left[\begin{array}{ccc}
\hat{\kappa}_{\|} & 0 & 0 \\
0 & \hat{\kappa}_{\perp} & 0 \\
0 & 0 & \hat{\kappa}_{\perp}
\end{array}\right]
$$

In the more general case, the conductivity tensor is rotated through the matrix operation,

$$
\boldsymbol{\kappa}=\mathbf{R}^{\mathrm{T}} \hat{\boldsymbol{\kappa}} \mathbf{R},
$$

resulting in $\boldsymbol{\kappa}$, the conductivity matrix in the coordinate system of the simulation. The rotation matrix is $\mathbf{R}$, which can be a function of the cell or face index depending on the type of material. If the through-plane direction is defined by the unit vector $\hat{i}^{\prime}=x_{1} \hat{i}+y_{1} \hat{j}+z_{1} \hat{k}$. i.e. $x_{1}, y_{1}, z_{1}$ are the directional cosines of the through-plane direction $\hat{i}^{\prime}$, and if the in-plane direction is defined by the unit vectors $\hat{j}^{\prime}$ and $\hat{k}^{\prime}$ with $x_{2}, y_{2}, z_{2}$ and $x_{3}, y_{3}, z_{3}$ being the respective directional cosines, then the rotation matrix $\mathbf{R}$ defined as

$$
\mathbf{R}=\left[\begin{array}{lll}
\hat{i^{\prime}} \cdot \hat{i} & \hat{i^{\prime}} \cdot \hat{j} & \hat{i^{\prime}} \cdot \hat{k} \\
\hat{j^{\prime}} \cdot \hat{i} & \hat{j^{\prime}} \cdot \hat{j} & \hat{j^{\prime}} \cdot \hat{k} \\
\hat{k^{\prime}} \cdot \hat{i} & \hat{k^{\prime}} \cdot \hat{j} & \hat{k}^{\prime} \cdot \hat{k}
\end{array}\right]=\left[\begin{array}{lll}
x_{1} & y_{1} & z_{1} \\
x_{2} & y_{2} & z_{2} \\
x_{3} & y_{3} & z_{3}
\end{array}\right]
$$

In the current formulation, the in-plane directions are assumed to be orthogonal, such that $\hat{k}^{\prime}=\hat{i}^{\prime} \times \hat{j}^{\prime}$. The cartesian conductivity tensor is computed for each cell, and at each time step, based on the directional cosines of the material's principal axes. 


\section{III.E. Boundary Conditions}

Numerically, boundary conditions are specified either by directly defining the face boundary flux or by setting the value of variables in ghost cells, i.e., the neighboring cells at a boundary that are outside of the simulation domain. The number of required ghost cells depends on the numerical stencil of the scheme. In the current formulation, boundary conditions are specified in both ways, and only one ghost cell is used. Boundary conditions are required for the temperature and the pyrolysis gas mixture velocity. Boundaries are either permeable or impermeable. In the case that the boundary is permeable, then the boundary pressure is required so that the gas velocity may be evaluated using Darcy's law. For the thermal boundary conditions, there are four options available: adiabatic, isothermal, constant heat flux, and radiative heat flux. All boundary conditions can be prescribed through spatial and temporal functions. This allows for the ability to impose a time-varying or steady-sate flow solution as a boundary condition onto the material. For an adiabatic boundary condition, there is no heat flux across the boundary. Thus, at the wall,

$$
q_{w}=\left.\left(\kappa_{i j} \frac{\partial T}{\partial x_{j}} \hat{n}_{i}\right)\right|_{\mathrm{w}}=0
$$

This requires that the temperature gradient across the boundary is zero, and therefore, the temperature in the ghost cell is set to be the same as in the first interior cell at the boundary.

For an isothermal boundary condition, the temperature at the wall is imposed resulting in a non-zero heat flux at the wall and a conserved value of the wall enthalpy. Since the wall temperature is specified, the ghost cell temperature is set as $T_{\text {ghost }}=2 T_{\mathrm{w}}-T_{\text {interior }}$, where $T_{\text {interior }}$ is the cell-center temperature of the first interior cell neighboring the boundary and $T_{\mathrm{w}}$ is the specified boundary temperature.

The temperature gradient at the face centriods is computed by averaging the cell-centered temperature gradients computed using the Gauss-Green method presented in Sec. III.C. If there is only a single ghost cell, this method can not be used for computing the temperature gradients at the boundary faces since the cellcentered temperature gradients are not known for the ghost cells. Thus, another method for approximating the temperature gradients at the wall is required. This is done by assuming the temperature gradient at the wall is approximately equal to the temperature gradient at the first cell interior, such that

$$
\left.\frac{\partial T}{\partial x_{j}}\right|_{\mathrm{w}}=\left.\frac{\partial T}{\partial x_{j}}\right|_{\mathrm{i}},
$$

This has been found to be the simplest and most robust method. Another option is to approximate the temperature gradient at the wall using a finite difference approximation in the wall normal direction. The temperature gradient in Cartesian space is then computed by multiplying the normal temperature deriviative by the face grid metric Jacobian.

When the heat flux at the wall is specified, the boundary condition is straight-forward since the flux along the surface is specified directly and does not explicitly depend on properly setting the ghost cell values. Yet, depending on the details of the scheme implementation, the ghost cell temperature may be used, e.g., when computing derivatives. Nevertheless, it is easy to compute the ghost cell temperature from the specified heat flux. In the simpliest of cases, if the thermal conductivity is a scalar, then

$$
\frac{q_{w}}{\kappa}=\frac{T_{\text {ghost }}-T_{\text {interior }}}{\Delta x}
$$

where $\Delta x$ is the distance between the cell centroids of the neighboring interior and ghost cells at the boundary. When the thermal conductivity is anisotropic, however, the calculation is complicated by the dependence of the orthonormal temperature gradients. In this case, an assumption is made that there is negligable variation in the temperature along the wall and that the heat flux is dominated by the wall normal temperature gradient.

Lastly, the radiative equilibrium boundary condition is similar to the specified heat flux once the net conductive flux has been computed. The re-radiation term is computed using the wall temperature and the specified environment temperature. The energy balance at the surface can be expressed as:

$$
\alpha \dot{q}_{\text {rad }}-\epsilon\left(T_{\text {wall }}-T_{\infty}\right)^{4}-\dot{q}_{\text {cond }}=0
$$

In this case the user specifies the radiative heat flux $\dot{q}_{r a d}$, as well as the environment temperature $T_{\infty}$. The quantities $\alpha$ and $\epsilon$ represent the absorptivity and emissivity of the material respectively, which are 
again prescribed based on material characterization, and may vary as the material chars. In the current formulation, the surface energy balance neglects the convective heat transfer to the wall and the energy transfer out of the wall due to pyrolysis blowing.

\section{Software Architecture and Parallelization}

Using the Fortran 2003/08 standard, it is possible to design highly modular and easily extensible data structures in Fortran. This provides two advantages. First, users can easily add new physical models and numerical routines with minimum or no modification to the code base. Second, code reusability minimizes the introduction of errors, makes it easier to read and understand, and makes code verification through unit and/or integration testing possible. In addition, pre-processing and post-processing utilities can easily make use of the core data structures.

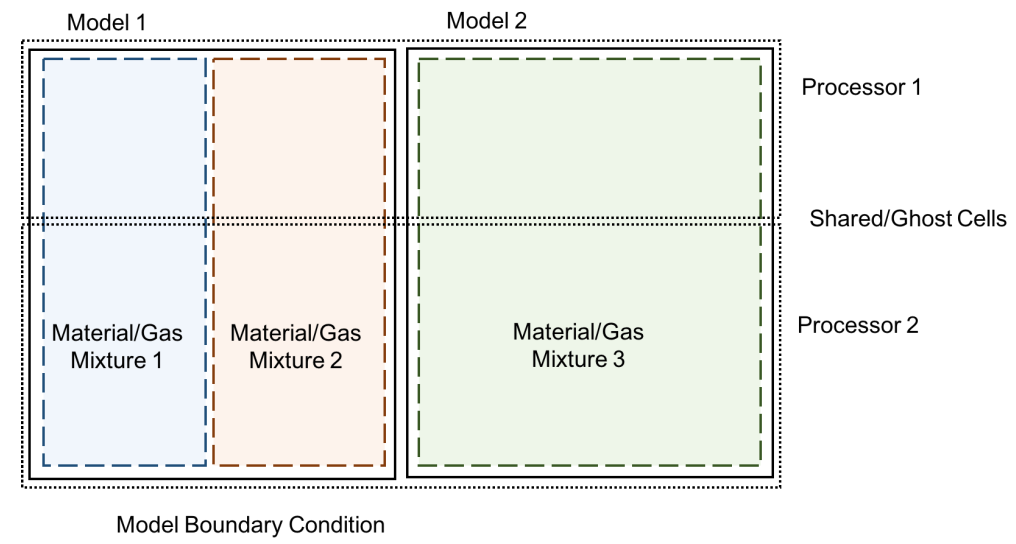

Figure 1. A simplisitc representation of how the data structures within the code are organized in order to simulate various material/gas mixtures, models, and physics using a modular approach.

At a high-level the design of the code divides the simulation into two main data groups as illustrated by Fig. 1. In the first group, a material and gas mixture zone defines the thermodynamic, transport, and chemical models to use at each computational point within the zone or group. In the second group, a model block specifies the set of governing equations to solve. The boundaries between these model blocks require the specification of additional boundary conditions since the primitive and conservative vectors are different between the two model blocks. In addition to this division, the entire domain can be partitioned into arbitrary number of processors.

Since the governing equations are solved using an unstructured approach, a careful ordering of the computational cells and faces within a processor allows for an efficient division of computation. Conservative fluxes are computed by looping over all faces within all blocks within a processor. The faces within each model block of a processor are ordered by interior zone faces, boundary zone faces, block boundary faces, and then shared boundary faces. This divides the computation into independent segments.

\section{Utilities}

\section{V.A. Mesh Deformation with Radial Basis Functions}

In order to numerically simulate the ablation of a material, some method to account for recession of the material surface is necessary. For instance, one can move the computational mesh directly or use a level-set approach to track the surface recession. In the current work, radial basis functions (RBF) are used to deform the computational mesh as the material recedes. This methodology ${ }^{9}$ as described by Rendall and Allen, ${ }^{10}$ is particularly well-suited for unstructured grids since it is indifferent to grid connectivity. Furthermore the methodology, when paired with a selective algorithm for determining control points, offers superior efficiency compared with linear elasticity methods.

Briefly, the radial basis function approach for mesh deformation is an interpolation scheme where the motion of the mesh points in the volume are driven by set of control points. For a material response model 
with an ablating surface, these control points are chosen to be the centroids of the face elements on the ablating surface. It is important to note, however, that the control points need not be a part of the mesh. This allows for some additional flexibility. Once the control points are selected, the motion of the grid points is determined by a weighted sum of the motion of the control points relative to the grid points, as in

$$
s(\mathbf{r})=\sum_{\mathbf{i}=\mathbf{1}}^{\mathbf{N}} \alpha_{\mathbf{i}} \phi\left(\| \mathbf{r}-\mathbf{r}_{\mathbf{i}}||\right),
$$

where $s(\mathbf{r})$ is the RBF interpolation, $\mathbf{r}$ is node displacement, $\phi\left(|| \mathbf{r}-\mathbf{r}_{\mathbf{i}}||\right)$ is the RBF evaluated at the distance between the node and control point $i$, and $\alpha_{i}$ is the weighting coefficient for that control point. The main computational cost of the method is dictated by the linear solve for the interpolation coefficients. The linear solve usually involves the inversion of a sparse linear system, but the sparseness is governed by the type of RBF support. Radial basis functions are grouped into those with global, local, and compact support, ${ }^{10}$ and those with global or local support are non-zero everywhere and result in dense matrices. Functions with compact support, however, decay to zero at a finite distance at the support radius and result in sparse matrices. For this reason, radial basis functions with compact support are most often used.

To demonstrate the implementation of the RBF approach, as well as show the influence of the support radius parameter, a simple test problem is presented using a $5 \mathrm{~cm} \times 10 \mathrm{~cm}$ diameter "puck" of material, pictured in Fig. 2. Five control points are selected on the top of the puck, and five are placed on the bottom at corresponding locations. The control points on the top of the geometry are displaced downwards by 2 $\mathrm{cm}$, whereas the control points on the bottom are held fixed. For this demonstration, the Wedland $\mathrm{C} 2$ radial basis function is used, which has a compact support. Figure 2 shows the resulting deformed surface mesh for three choices of the support radius, $R$. Increasing the support radius results in a broader deformation. For physical applications, the support radius, $R$ is set to a characteristic length scale of the problem. In this case, both $R=5 \mathrm{~cm}$ and $R=10 \mathrm{~cm}$ would be representative of typical choices, however, a choice of $R=10 \mathrm{~cm}$ results in a more uniform recession of the top geometry. Adding more control points would reduce the dependence of the deformation of the support radius, but this example highlights that a reduced set of control points is sufficient for capturing a deformation.

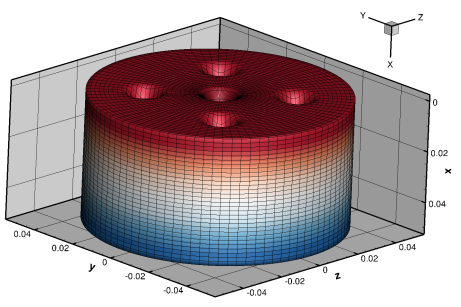

(a) $R=1 \mathrm{~cm}$

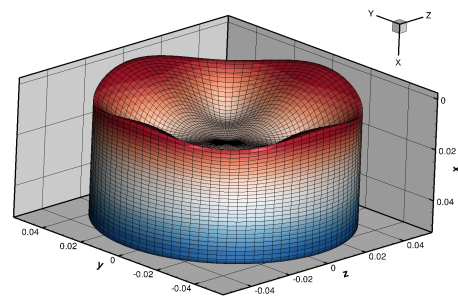

(b) $R=5 \mathrm{~cm}$

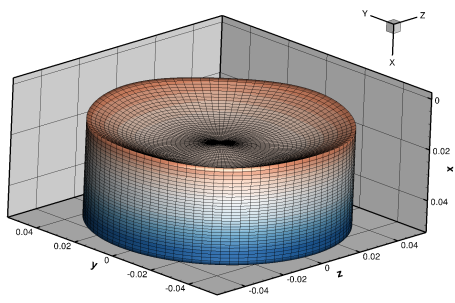

(c) $R=10 \mathrm{~cm}$

Figure 2. Radial basis function based mesh deformation for various choices of support radius

Implementing this within the material response model requires adding additional numerical fluxes to account for the motion of the computational mesh. Fully incorporating these fluxes into the time-dependent materal response is a part of the on-going development of the solver. Currently, all mesh deformation is done independently of the time advancement scheme.

\section{V.B. Equilibrium Calculations and Material Database}

A choice of three equilibrium chemistry solvers is provided. The first equilibrium solver is an updated version of the NASA Chemical Equilibrium with Applications (CEA) code, ${ }^{4}$ which has been adapted in order to be fully integrated into the current solver. The additional two options incorporate equilibrium solvers from separate library packages that are often used within the aerospace community for evaulating thermodynamic and chemical properties of mixtures. The first is the MUlti-component Thermodynamic And Transport properties for IONized gases in $\mathrm{C}^{++}\left(\mathrm{MUTATION}^{++}\right) \operatorname{code}^{11}$ developed at the VonKarman Institute (VKI), and the second the Cantera library package. ${ }^{12}$

The equilibrium state of a gas mixture is computed by minimizing the mixture Gibbs free energy. Numerically, the NASA CEA code ${ }^{4}$ uses a Newton-Raphson iteration scheme to converge to the equilibrium 

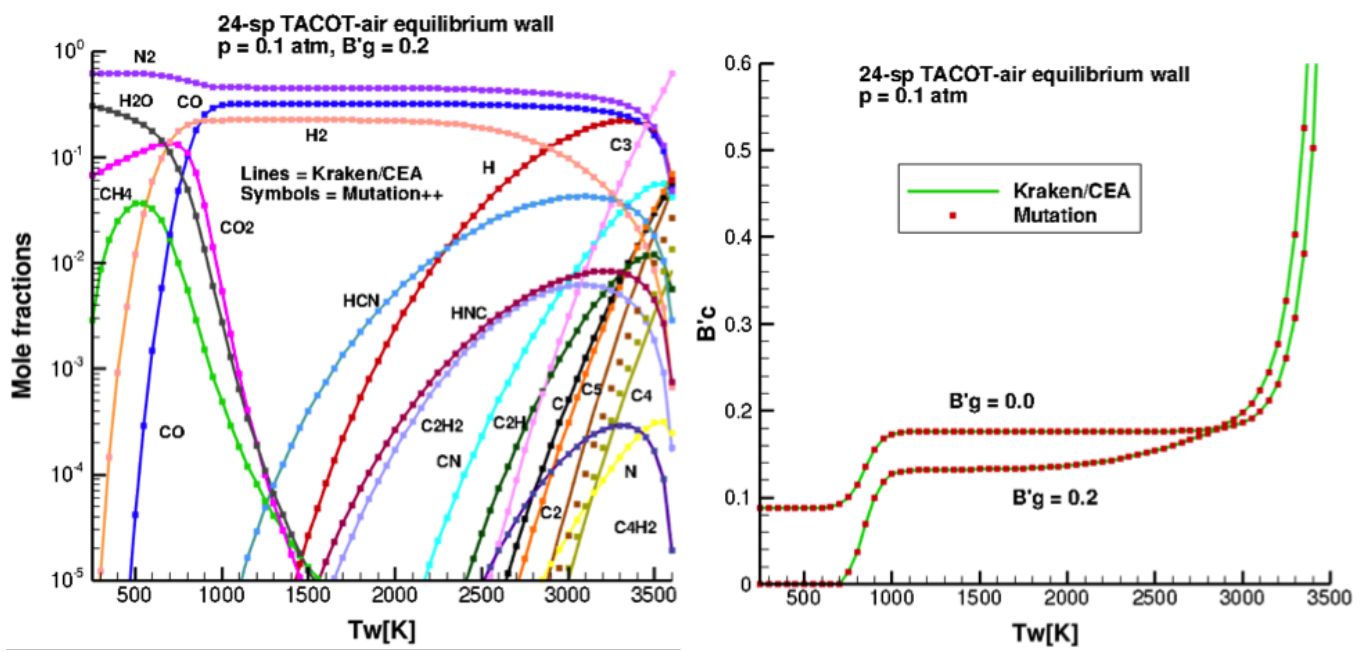

Figure 3. Comparison between in-code CEA solver, and Mutation++ library for the 24 species TACOT-air system.

state, while the Mutation++ code solves the equilibrium equations using a technique known as Gibbs function continuation, which is a continuation-Krylov iterative scheme and is claimed to be more robust than standard Newton-Raphson method. ${ }^{11}$ Both codes determine whether to include or exclude condensed species based on whether doing so will decrease mixture Gibbs free energy.

Since the material and gas mixtures are in chemical and thermal equilibrium, the solver behavior is based on predefined gas mixtures. The gas mixtures can include condensed species and can represent either pure gas mixtures or or gas-material systems. Figure 3 shows species mole fractions computed by the two codes for a mixture of air and the Theoretical Ablative Composite for Open Testing (TACOT) ${ }^{13}$ solid material, which totals an equilibrium mixture of 24 species. The pressure is $0.1 \mathrm{~atm}$ and the normalized pyrolysis gas mass flux $\left(B_{g}^{\prime}\right)$ is 0.2 . The species mole fractions for both the Mutation++ library and the NASA CEA routines are similar over the entire temperature range except for $\mathrm{C}_{4}$ and $\mathrm{C}_{5}$ at higher temperatures. The reason for this discrepancy is that Mutation++ uses modified polynomial curve fits for C4 and C5. Additionally, equilibrium solvers are often used to compute the normalized char mass flux $\left(B_{c}^{\prime}\right)$, which is an important quantity in computing the surface energy balance for an ablating material. Figure $3 \mathrm{~b}$ shows $B_{c}^{\prime}$ as a function of temperature for two values of normalized pyrolysis gas mass flux at a pressure of $0.1 \mathrm{~atm}$.

An important part of any material response model is ability to easily define the properties of the material and product gases. For this reason, the inputs for the material database are flexible. All material properties, such as the thermal conductivity tensor or the specific heat can be specified as either general polynomial functions with linear coefficients or as a tabulated data. In both cases, the properties can be specified as temperature and/or pressure dependent values. The material property database information can be accessed or generated using a standalone utility.

\section{Results}

\section{VI.A. Verification to Analytical Solutions}

There are several analytical solutions to the time dependent heat conduction equation. Assuming there is no pyrolysis and that the material properties are isotropic, comparisons can be made to these analytical solutions to assess the order of accuracy of the code. All of the following studies use a rectangular computational grid with a length, $L_{x}=1$ meter and cross-sectional area, $L_{y} \times L_{z}$, of 0.1 meter $\times 0.1$ meter. The boundaries at $x=0$ nd $x=L_{x}$ are varied in each case, but the boundaries in the $y$ and $z$ directions are always assumed to be adiabatic walls. The domain is decomposed with hexahedral and tetrahedral elements of varying degrees of refinement. The material properties are normalized to unity, and in one case are linearly dependent on the temperature such that they vary from 0 to 1 . The root-mean-square error is computed as follows:

$$
\mathrm{RMS}=\sqrt{\frac{\sum_{\mathrm{i}=1}^{\mathrm{N}_{\mathrm{c}}}\left(T_{\text {analytical }, \mathrm{i}}(t)-T_{\text {numerical }, \mathrm{i}}(t)\right)^{2}}{\mathrm{~N}_{\mathrm{c}}}}
$$


where $\mathrm{N}_{\mathrm{c}}$ is the total number of computational cells.

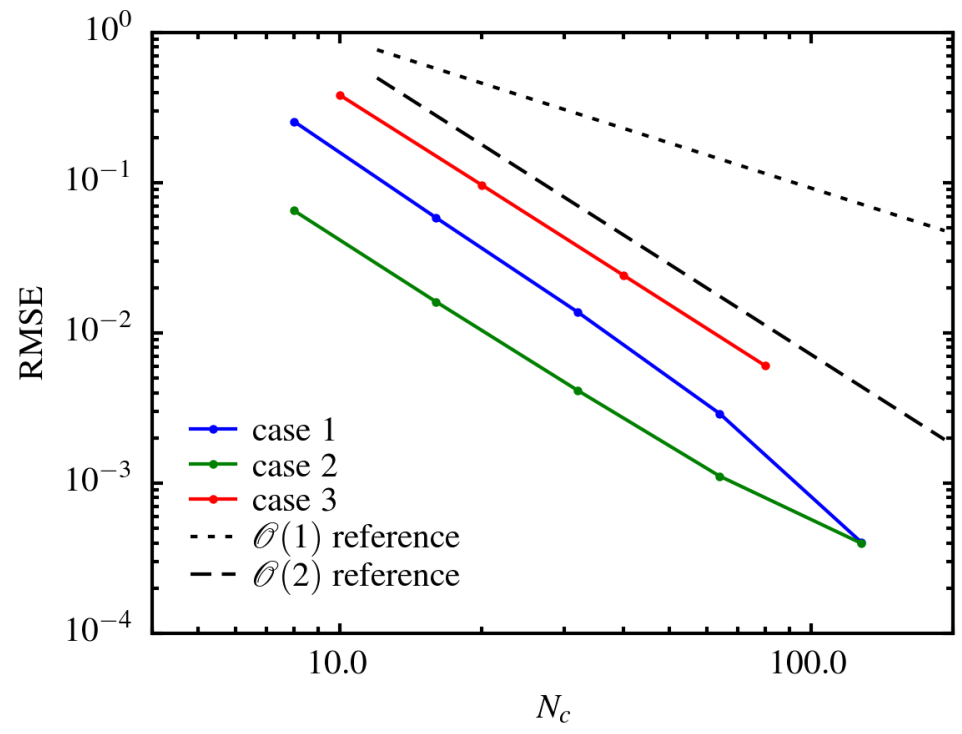

Figure 4. Order of convergence for a steady-state quasi one-dimensional thermal conduction simulation. The normalized root-mean-square error is determined by computing the differences in the temperature at each $x$-location with reference to the analytical solution.

For case 1 , the surfaces at $x=0$ and $x=L_{x}$ are defined by a Dirichlet boundary condition, i.e., isothermal wall. The temperature at each surface is $300 \mathrm{~K}$ and $400 \mathrm{~K}$, respectively, and the thermal conductivity of the material is constant. Normalizing time dependent temperature within the domain results in the analytical solution

$$
\frac{T(x=0)-T(t)}{T(x=0)-T\left(x=L_{x}\right)}=2 \sum_{n=0}^{\infty} \frac{(-1)^{n}}{\left(n+\frac{1}{2}\right) \pi} \exp \left[-\left(n+\frac{1}{2} \pi\right)^{2} \frac{\alpha t}{L_{x}^{2}}\right] \cos \left(\left(n+\frac{1}{2}\right) \frac{\pi x}{L_{x}}\right) .
$$

where the thermal diffusivity is $\alpha=\kappa / \rho c_{p}$.

For cases 2 and 3, the surfaces at $x=0$ and $x=L_{x}$ are prescribed Neumann boundary conditions. At $x=0$, a constant heat flux wall of $\dot{q}_{w}$ is specified. At $x=L_{x}$ the surface is adiabatic, corresponding to a heat flux of zero. For case 2, the material properties are constant for case 2 , but in case 3 , the thermal conductivity and specific heat are taken to be linear functions of the temperature. The analytical solutions for both cases are self-similar. For the linear dependent material properties, let $T_{1}$ define the temperature at the lower bound, and let $T_{2}$ define the temperature at the upper bound. Similarly, $\kappa_{1}$ and $\kappa_{2}$ are the thermal conductivities evaluated at the lower and upper bound temperatures. Using the definition,

$$
\Theta=\left(T-T_{1}\right)+\frac{\kappa_{2}-\kappa_{1}}{T_{2}-T_{1}} \frac{1}{2 \kappa_{1}}\left(T-T_{1}\right)^{2}
$$

the solution to the heat conduction equation, normalized by the heat flux, is given by

$$
\frac{\theta(x, t)-\theta(x=0)}{\dot{q}_{w} L_{x} / \kappa_{1}}=\frac{\alpha t}{L_{x}^{2}}+\frac{1}{3}-\frac{x}{L}+\frac{1}{2}\left(\frac{x}{L}\right)^{2}-\frac{2}{\pi^{2}} \sum_{n=1}^{\infty} \frac{1}{n^{2}} \exp \left[-n^{2} \pi^{2} \frac{\alpha t}{L_{x}^{2}}\right] \cos \left(n \pi \frac{x}{L_{x}}\right)
$$

where in this case, $\dot{q}_{w}=7.5 \times 10^{5} \mathrm{~W} \cdot \mathrm{m}^{-2}$. For case $2, \theta(x, t)=T(x, t)$ since $\kappa=\kappa_{1}=\kappa_{2}$. For case 3 , the thermal conductivity is defined as

$$
\kappa(T)=\kappa_{1}+\frac{\kappa_{2}-\kappa_{1}}{T_{2}-T_{1}}\left(T-T_{1}\right)
$$

A similar linear function defines the specific heat and the thermal diffusivity.

For case 1, 2, and 3, the order of convergence is displayed in Fig. 4. As expected, the numerical scheme converges to second order for each case. 
Case 4 is similar to case 2, but the heat flux at the $x=0$ surface is sinusoidal time-varying function, $\dot{q}_{w}(t)=\dot{q}_{w, 0} \cos \omega t$, where $\omega$ is the frequency of oscillation. For the results shown here, $\omega=\pi \sec ^{-1}$ and $\dot{q}_{w, 0}=1.0 \times 10^{4} \mathrm{~W} \cdot \mathrm{m}^{-2}$. The test is useful, but since an analytical solution exists only for semi-infinite domains, it is difficult to calculate the error directly. Numerically, a semi-infinite domain is modeled by reducing the diffusivity of the material relative to the length of the domain such that there is a large region near the $x=L_{x}$ surface that remains at an approximately constant temperature. This is possible since the applied heat flux changes sign. Figure 5 shows the temperature profile at an instance in time.

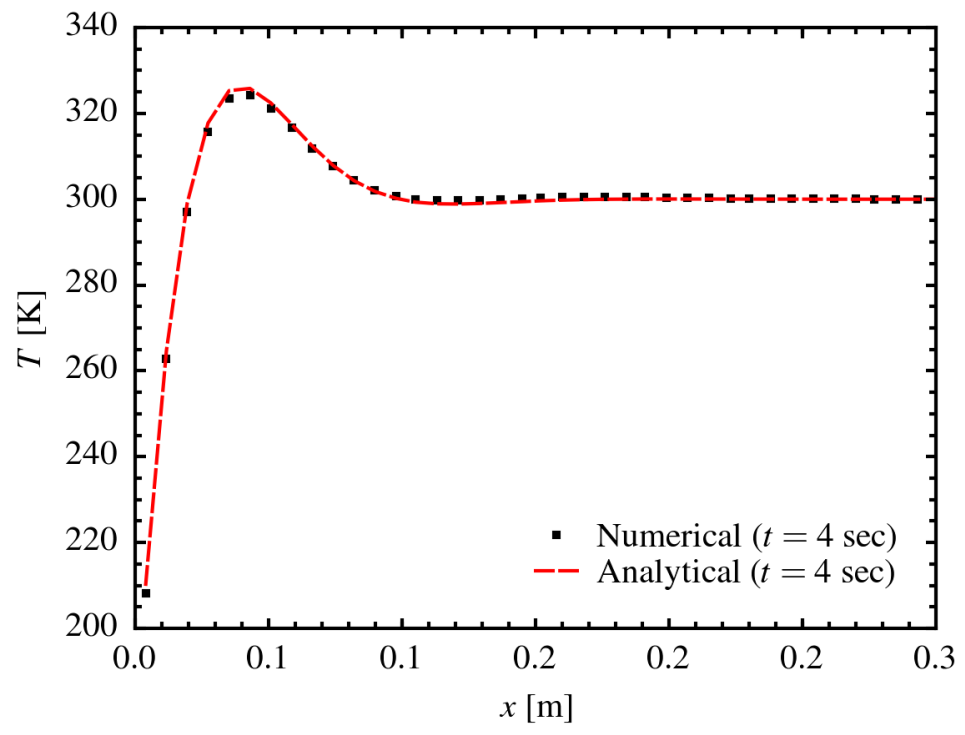

Figure 5. The in-depth temperature of a constant property material corresponding to a time-varying sinusoidal heat flux applied at $x=0 \mathrm{~m}$ with a rate and magnitude of $\omega=\pi \sec ^{-1}$ and $\dot{q}_{w, 0}=1.0 \times 10^{4} \mathrm{~W} \cdot \mathbf{m}^{-2}, \mathbf{r e s p e c t i v e l y}$.

\section{VI.B. Verification to Test Problems}

Over several years, there has been an effort to establish a set of common verification tests within the material ablation community in order to understand the variances between the physical models and numerical methods of material response models developed at various government agencies and universities. The test series begins with simple tests focusing on one-dimensional in-depth material response and increases in complexity to account for multi-dimensional effects as well as chemical and thermal non-equilibrium.

The first test establishes a baseline by designing a test for the traditional material response models like the Charring material ablation $(\mathrm{CMA})^{2}$ and Fully Implicit Ablation and Thermal (FIAT). ${ }^{14}$ This test verifies the physics of heat conduction, pyrolysis, and simplified mass transfer in a one-dimensional domain of $5 \mathrm{~cm}$ composed of the Theoretical Ablative Composite for Open Testing (TACOT) material. ${ }^{13}$ All boundaries are adiabatic except a single isothermal wall at temperature of $1644 \mathrm{~K}$ that heats the material for the duration of 1 second. The initial pressure and temperature are $101325 \mathrm{~Pa}$ and $298 \mathrm{~K}$, resepectively. Figure 6 shows the transient temperature profiles at the in-depth locations, $x=1 \mathrm{~mm}, 2 \mathrm{~mm}, 4 \mathrm{~mm}, 8 \mathrm{~mm}, 16 \mathrm{~mm}$, and $50 \mathrm{~mm}$. The results are compared to the one-dimensional Fully Implicit Ablation and Thermal response program (FIAT),${ }^{14}$ a material response code developed at NASA's Ames Research Center. The percent error difference between the two codes is less than 3 precent.

In a second test, the same one-dimensional domain, but instead composed of Phenolic Impregnated Carbon Ablator (PICA) material, is subjected to a radiative heating pulse of $20 \mathrm{~W} \cdot \mathrm{cm}^{-2}$ for 20 seconds on one side of the domain. Figure 7 once again shows the transient temperature profiles at the in-depth locations, $x=1 \mathrm{~mm}, 2 \mathrm{~mm}, 4 \mathrm{~mm}, 8 \mathrm{~mm}, 16 \mathrm{~mm}$, and $50 \mathrm{~mm}$, and the results are once again compared to FIAT.

In the last test, a qualitative demonstration of the three-dimensional and unstructured characteristics of the solver is provided using a simplified version of third ablation workshop test case. In this case, an Iso-Q sample, typical of most arc-jet conditions ${ }^{15,16}$ is simulated. The test case is not meant to be representative since ablation is not accounted for in the simulation, but the test case does serve as a baseline for code to code 


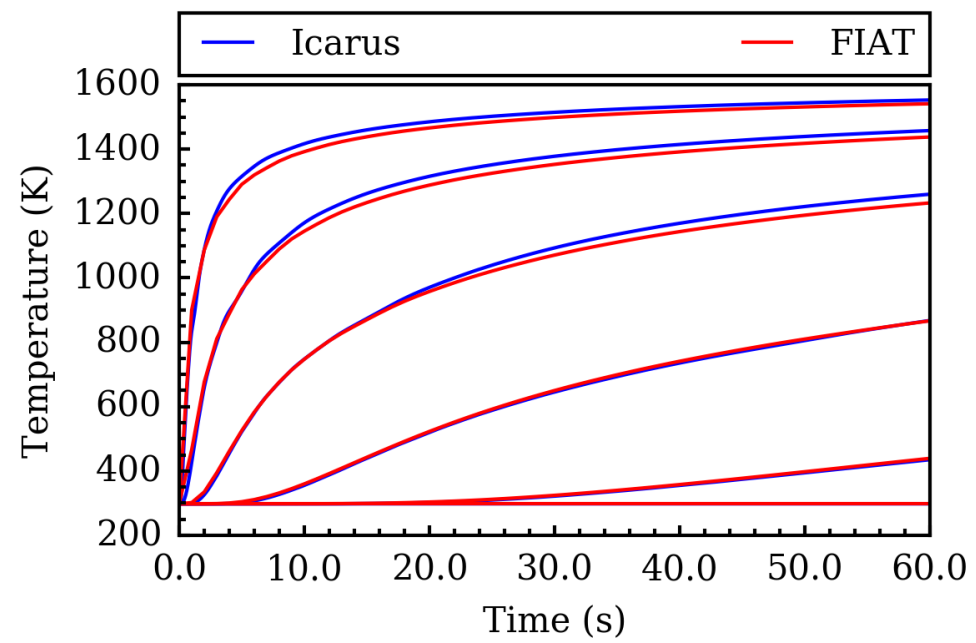

Figure 6. Transient temperature profiles at $x=1 \mathrm{~mm}, 2 \mathrm{~mm}, 4 \mathrm{~mm}, 8 \mathrm{~mm}, 16 \mathrm{~mm}$, and $50 \mathrm{~mm}$ with comparison to results computed using the code FIAT for the first ablation workshop test case.

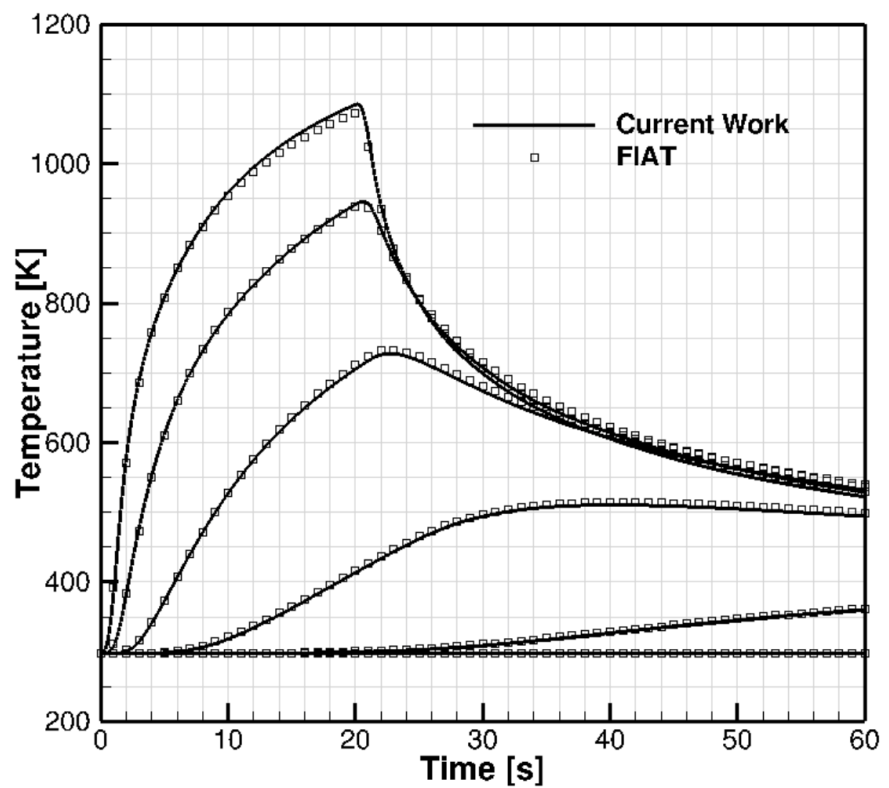

Figure 7. Transient temperature profiles at $x=1 \mathrm{~mm}, 2 \mathrm{~mm}, 4 \mathrm{~mm}, 8 \mathrm{~mm}, 16 \mathrm{~mm}$, and $50 \mathrm{~mm}$ with comparison to results computed using the code FIAT. The profiles are such that the peak temperature is largest at $x=1$ mm and smallest at $x=50 \mathrm{~mm}$. 
comparisons. The material of the Iso-Q sample is TACOT, and the sample has a outside cylindrical radius of $50 \mathrm{~mm}$, and the surface ellipse on the top of the cylinder has a major axis of $50 \mathrm{~mm}$ and a minor axis of 13.3397. This case demonstrates the ability to model three-dimensional material response using unstructured elements, as seen in Fig. 8(b). The time history of the temperature is compared to the CHarring Ablator Response (CHAR) code. ${ }^{17}$

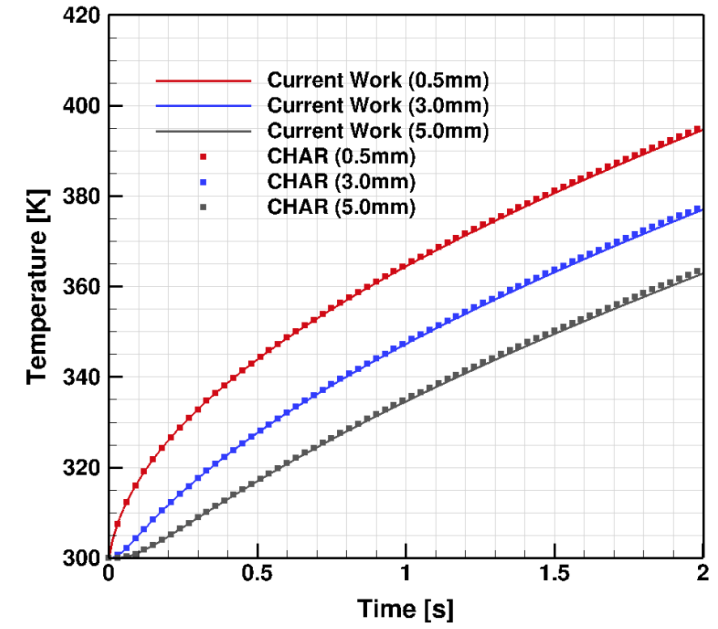

(a)

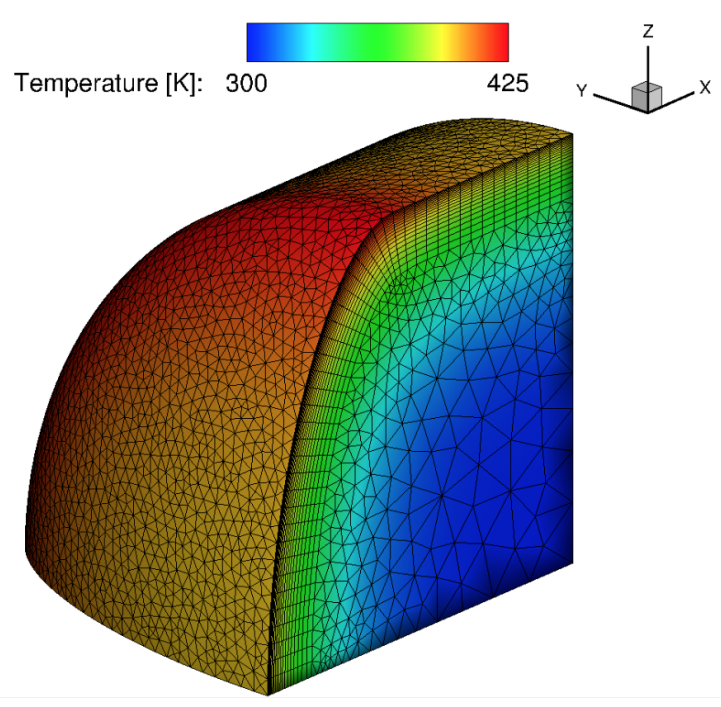

(b)

Figure 8. (a) Transient temperature profiles at three in-depth locations, $x=0.5 \mathrm{~mm}, 3.0 \mathrm{~mm}$, and $5.0 \mathrm{~mm}$ with comparison to results computed using the code CHAR. (b) Temperature contours at $t=2$ seconds.

\section{Conclusions and Future Work}

Using a preliminary set of verification tests, Icarus, a new three-dimensional, unstructured material response model, shows good agreement to results obtained from existing simulation tools, FIAT ${ }^{14}$ and CHAR.${ }^{17}$ The favorable comparisons verify the numerical implementation, and convergence studies show that the numerical model obtains second-order accuracy for a simple thermal conduction problem. Additionally, the unstructured formulation of the model allows for the simulation of the material response in complex bodies. In addition to these preliminary results, there are several on-going tasks that will be presented in the future work. With the verification of the baseline phsyics of heat conduction and pyrolysis, higher fidelity physical models will be implemented. In particular, this will include non-equilibrium pyrolysis chemistry. Additionally, the current assumption of thermal and chemical equilibrium at the surface boundary can be relaxed to include interaction with the gas and potential melt flow or spallation. As these higher-fidelity models are incorporated, additional features useful in the design of TSP materials will be added.

\section{Acknowledgements}

This work has been sponsored by NASA's Entry Systems Modeling project and NASA's Asteroid Threat Assessment Project. The authors Joseph Schulz, Suman Muppidi, and Grant Palmer are supported through the NNA15BB15C contract bewteen NASA Ames Research Center and AMA, Inc.

\section{References}

\footnotetext{
${ }^{1}$ Lachaud, J., van Eekeln, T., Scoggins, J., Magin, T., and Mansour, N., "Detailed chemical equilibrium model for porous ablative materials," International Journal of Heat and Mass Transfer, Vol. 90, 2015, pp. 1034-1045.

${ }^{2}$ Moyer, C. and Rindal, R., "An Analysis of the Coupled Chemically Reaction Boundary Layer and Charring Ablator, Part II, Finite Difference Solution for the In-Depth Response of Charring Materials Considering Surface Chemical and Energy
} 
Balances," NASA CR-1061, June 1968, pp. 1-168.

${ }^{3}$ Amar, A., Blackwell, B., and Edwards, J., "Development and Verification of a One-dimensional Ablation Code Including Pyrolysis Gas Flow," Journal of Thermophysics and Heat Transfer, Vol. 23, No. 1, 2009, pp. 59-71.

${ }^{4}$ McBride, B. J. and Gordon, S., Computer Program for Calculation of Complex Chemical Equilibrium Compositions and Applications, NASA Lewis Resarch Center, June 1996.

${ }^{5}$ Gottlieb, S. and Shu, C.-W., "Total variation diminishing Runge-Kutta schemes," Mathematics of Computation, Vol. 67, 1998, pp. 73-85.

${ }^{6}$ Ruuth, S. and Hundsdorfer, W., "High-order linear multistep methods with general monotonicity and boudedness properties," Journal of Computational Physics, Vol. 209, No. 17, 2005, pp. 226-248.

${ }^{7} \mathrm{Shu}$, C., "Total-variation diminishing time discretizations," SIAM Journal on Scientific and Statistical Computing, Vol. 9, 1988, pp. 1073-1084.

${ }^{8}$ Nompelis, I., Drayna, T., and Candler, G., "A Parallel Unstructured Implicit Solver for Hypersonic Reacting Flow Simulation," 17th AIAA Computational Fluid Dynamics Conference, June 2005, pp. 1-17.

${ }^{9}$ de Boer, A., van der Schoot, M., and Bijl, H., "Mesh deformation based on radial basis function interpolation," Computers \& Structures, Vol. 85, No. 11-14, 2007, pp. 784-795.

${ }^{10}$ Rendall, T. and Allen, C., "Efficient mesh motion using radial basis functions with data reduction algorithms," Journal of Computational Physics, Vol. 228, No. 17, 2009, pp. 6231-6249.

${ }^{11}$ Development of Mutation++: Multicomponent Thermodynamic and Transport Properties for Ionized Plasmas written in $\mathrm{C}++$, Reston, Virginia, June 2014, American Institute of Aeronautics and Astronautics.

${ }^{12}$ Goodwin, D. G., Moffat, H. K., and Speth, R. L., "Cantera: An Object-oriented Software Toolkit for Chemical Kinetics, Thermodynamics, and Transport Processes," http://www.cantera.org, 2016, Version 2.2.1.

${ }^{13}$ Lachaud, J., Eekelen, T., Bianchi, D., and Martin, A., "TACOT : Theoretical Ablative Composite for Open Testing," http://dx.doi.org/10.13023/ablation.test.TACOT, 2014, Version 3.0.

${ }^{14}$ Chen, Y. and Milos, F. S., "Ablation and Thermal Response Program for Spacecraft Heatshield Analysis," Journal of Spacecraft and Rockets, Vol. 36, No. 3, 1999, pp. 475-483.

${ }^{15}$ Milos, F. S. and Chen, Y. K., "Two-Dimensional Ablation, Thermal Response, and Sizing Program for Pyrolyzing Ablators," Journal of Spacecraft and Rockets, Vol. 46, No. 6, Nov. 2009, pp. 1089-1099.

${ }^{16}$ Ablation Workshop Test Case Series 3, 5th Ablation Workshop, Feb. 28 - Mar. 12014.

${ }^{17}$ Amar, A. J., Calvert, N., and Kirk, B., "Development and Verification of the Charring Ablating Thermal Protection Implicit System Solver," 49th AIAA Aerospace Sciences Meeting including the New Horizons Forum and Aerospace Exposition, Dec. 2010, pp. 1-57. 